\title{
Efeitos dos Termos Individuais do Potencial Gravitacional Lunar no Movimento de Satélites Artificiais ao Redor da Superfície da Lua
}

\author{
Liana Dias Gonçalves, Evandro Marconi Rocco, \\ Instituto Nacional de Pesquisas Espaciais- INPE \\ 12227-010, São José dos Campos, SP, Brasil \\ E-mails: lianadgon@gmail.com, evandro.rocco@inpe.br
}

\author{
Rodolpho Vilhena de Moraes \\ Universidade Federal de São Paulo - UNIFESP \\ 12231-289, São José dos Campos, SP, Brasil \\ E-mails: rodolpho.vilhena@gmail.com
}

Resumo: O presente trabalho analisa a influência da não homogeneidade do campo gravitacional lunar na órbita de um satélite artificial. O modelo baseia-se nos harmônicos esféricos, de acordo com o desenvolvimento apresentado por Konopliv. Este modelo nos permite considerar harmônicos esféricos até grau e ordem 100. Simulações são realizadas para que sejam feitas as seguintes análises: a contribuição de cada termo do potencial gravitacional lunar, a variação da perturbação devido à inclinação da órbita e a atuação do sistema de controle. Manobras de transferência e de correção de satélites lunares são simuladas considerando empuxo contínuo e controle de trajetória em malha fechada.

\section{Introdução}

O comportamento de um satélite artificial em órbita lunar é perturbado, principalmente, pela não homogeneidade do campo gravitacional da Lua, o que tende a causar variações nos elementos orbitais do satélite artificial.

Considerando-se o longo período de atividade da missão espacial, é necessária a realização de manobras de manutenção orbital para manter o satélite em uma órbita pré determinada.

Para o estudo das perturbações devido a não esfericidade do campo gravitacional lunar, o modelo LP100K (Konopliv et al., 2001) foi inserido num simulador de trajetória (STRS), desenvolvido por Rocco (2008). Este modelo permite estudar a influência do grau e ordem dos harmônicos esféricos gravitacionais na órbita de um satélite artificial lunar com precisão máxima de grau e ordem 100. Estudos e análises de tal influência, bem como simulações considerando a perturbação devido ao potencial gravitacional lunar na órbita de um satélite artificial, podem ser encontradas em Gonçalves (2013).

O STRS permite controlar a trajetória de modo que os desvios e erros nas variáveis de estado sejam minimizados. Para realizar as manobra de correção de trajetória, o STRS utiliza sistema de propulsão contínua e controle de trajetória em malha fechada. Uma descrição mais detalhada do controlador de trajetória orbital podem ser encontradas em Rocco (2012).

\section{Potencial gravitacional lunar}

O potencial gravitacional da Lua é expresso pelos coeficientes dos harmônicos esféricos normalizados, dado pela equação (1) (Konopliv et al., 2001; Kuga, 2011):

$$
U(r, \lambda, \phi)=\frac{\mu}{r}++\frac{\mu}{r} \sum_{n=2}^{\infty} \sum_{m=0}^{n}\left(\frac{a_{e}}{r}\right)^{n}\left(\bar{C}_{n m} \cos m \lambda+\bar{S}_{n m} \operatorname{sen} m \lambda\right) \bar{P}_{n m}(\operatorname{sen} \phi)
$$


em que: $n$ é o grau e $m$ é a ordem, $\mu$ é a constante gravitacional e $r$ é o raio lunar equatorial. $\bar{P}_{n m}$ são os polinômios associados de Legendre normalizados. $a_{e}$ é o raio vetor (distância), $\phi$ é a latitude e $\lambda$ é a longitude.

\section{O modelo LP100K}

O campo gravitacional lunar foi determinado utilizando dados de diferentes missões lunares já realizadas.

Os dados para o desenvolvimento do modelo LP100K foram obtidos por uma das mais importantes missões, a Lunar Prospector (1998-1999), que é a terceira missão do Discovery, programa de exploração da NASA, que realizou a primeira medição do campo gravitacional da Lua. Desde a época do desenvolvimento do modelo LP100K, usando a missão Lunar Prospector, não houve observações diretas do lado oculto da Lua. O conhecimento sobre o campo gravitacional lunar foi obtido a partir de efeitos a longo prazo observados na órbita de satélites artificiais.

O LP100K modelo apresentado por Konopliv é uma representação dos harmônicos esféricos devido à gravidade planetária baseada no potencial gravitacional do corpo celeste, dado pela equação (1).

A saída calculada pelo modelo fornece as componentes $\mathrm{x}, \mathrm{y}$ e $\mathrm{z}$ para a aceleração da gravidade a cada instante de tempo ao longo da órbita de um satélite artificial. É possível considerar os harmônicos esféricos até grau e ordem 100. Por uma comparação entre a aceleração da gravidade de um campo central e a aceleração gravitacional fornecida pelo modelo da Konopliv, obtém-se o incremento de velocidade perturbador sobre o satélite, sendo possível, por meio do problema inverso, a obtenção dos elementos keplerianos que caracterizam a órbita de um do satélite artificial, a fim de que seja feita uma análise do movimento orbital.

\section{Simulações e resultados}

Uma vez que o objetivo deste trabalho é analisar a influência do potencial gravitacional em uma órbita lunar de um satélite artificial, foram realizados três estudos utilizando simulações feitas no STRS, conforme descrito abaixo.

O primeiro estudo visa analisar a influência de cada termo do potencial gravitacional na órbita de um satélite artificial. Essa tarefa foi feita de forma indireta, obtendo-se os valores dos incrementos de velocidade aplicados por $N$ termos do potencial durante uma órbita do veículo espacial e, em seguida, repete-se esse cálculo para $N+1$ termos. Fazendo a diferença entre os incrementos de velocidade, obtém-se a contribuição da adição de mais termos ao potencial.

Em seguida, o estudo é feito com o objetivo de variar a inclinação, de modo a mostrar a influência da perturbação devido ao potencia gravitacional lunar neste elemento orbital.

A situação em que a variação dos elementos orbitais é usada para minimizar o consumo de combustível para a manutenção orbital é usual. No entanto, o objetivo aqui é medir os efeitos da adição de cada termo do potencial e não encontrar a estratégia que minimiza o consumo de combustível. Uma idéia similar foi feita por Prado (2013) para uma nave espacial que viaja ao redor da Terra e é perturbada pela Lua e pelo Sol.

Após a análise da influência de cada termo do potencial gravitacional lunar, bem como a influência para diferentes valores de inclinação, quatro simulações orbitais são realizadas.

Foram analisados os casos em que apenas manobras de correção são realizadas, e o caso em que manobras de transferências e manobras de correção são realizadas. Com respeito às manobras de transferência, foi simulado um caso para transferir o satélite a partir de uma órbita lunar alta para uma órbita lunar baixa, e um caso para transferir o satélite a partir de uma órbita lunar baixa para uma órbita lunar alta. 


\subsection{Incremento de velocidade}

Começaremos o estudo, analisando a importância de cada termo do potencial gravitacional lunar.

Esta tarefa é repetida 100 vezes, cada vez adicionando o próximo termo do potencial gravitacional, o que significa que para cada termo do potencial gravitacional lunar, uma simulação é feita durante o período de uma órbita. Neste momento, em todas as simulações o sistema de controle não foi acionado, uma vez que o objetivo é analisar os efeitos da perturbação devido ao potencial gravitacional na órbita do satélite .

Assim, a variação total da velocidade aplicado ao satélite devido aos termos perturbadores é obtida, assumindo que o movimento é influenciado apenas pelo potencial da Lua de acordo com o modelo do Konopliv. Esta medida dá o efeito da adição de um novo elemento para o potencial gravitacional da Lua, e não a contribuição de cada termo individualmente. Então, após estas simulações, a subtração da contribuição dos termos anteriores mostra como a adição de um novo termo muda as características orbitais.

A Figura 1 mostra a variação total da velocidade decorrente da não homogeneidade da distribuição de massa lunar, com a dinâmica que leva em conta $N+1$ termos do potencial gravitacional menos a mesma variação levando em conta $\mathrm{N}$ termos do potencial gravitacional.

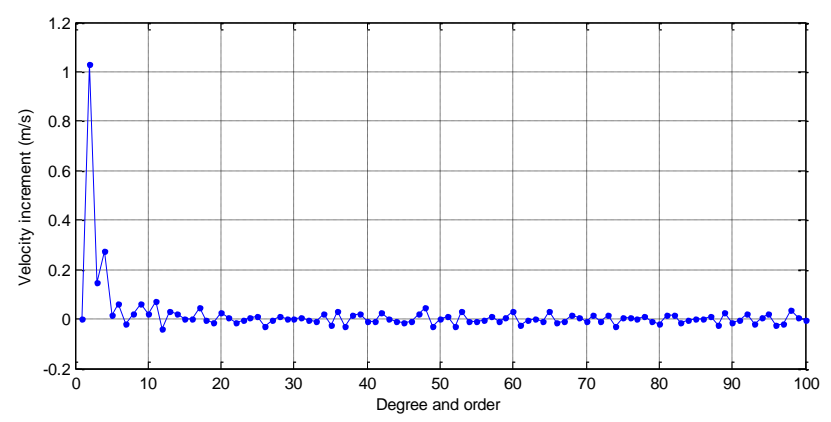

Figura 1: Diferença entre a variação de velocidade aplicada ao veículo espacial com $N+1$ e com $N$ termos do potencial lunar

As condições iniciais da órbita são: semi-eixo maior: $1900000 \mathrm{~m}$, excentricidade: 0.001, inclinação: $45^{\circ}$, ascensão reta do nodo ascendente: $20^{\circ}$, argumento do periapside: $100^{\circ}$, anomalia média: $1^{\circ}$.

Nota-se um esperado domínio do primeiro termo do potencial perturbador, tal como observado em Carvalho et al. (2008), Carvalho et al. (2009(a)), Carvalho et al. (2009(b)) e Carvalho et al. 2010. No entanto, também é possível ver que os resultados dos termos seguintes não são monotonicamente decrescentes, o que significa que um termo pode ser de ordem mais elevada, e proporcionar uma contribuição significativa para o aumento da velocidade do satélite, como estudado por Gonçalves (2013). Um estudo semelhante pode ser encontrado em Ramanan (2005).

Termos negativos aparecem na Figura 1, o que significa que a adição deste termo no potencial gravitacional lunar diminui a perturbação aplicada ao satélite, de modo a reduzir a variação de velocidade necessária para a manutenção de uma órbita kepleriana. Em outras palavras, este termo ajuda a controlar o satélite artificial, porque o seu efeito é oposto à resultante das condições anteriores, ou seja, a favor do sistema de controle.

\subsection{Inclinação}

Como visto na Seção 4.1, cada termo do potencial gravitacional lunar provoca uma influência diferente sobre a órbita de um satélite artificial. 
O objetivo agora é analisar o efeito da perturbação na inclinação do satélite artificial lunar. Um estudo de um satélite artificial perturbado pelo potencial gravitacional para o caso da inclinação crítica é realizada em Carvalho (2009(b)) e Carvalho (2010).

Para executar esta tarefa, os valores de incremento de velocidade de um satélite artificial lunar devido ao potencial perturbador foram obtidos com base no modelo apresentado por Konopliv. Para evitar uma quantidade excessiva de resultados, as simulações foram feitas considerando os termos até grau e ordem 2, 10, 50 e 100. Os resultados são mostrados na Figura. 2.

Órbitas foram analisadas com os seguintes valores de inclinação : $0^{\circ}, 15^{\circ}, 30^{\circ}, 45^{\circ}, 60^{\circ}, 75^{\circ}$, $90^{\circ}, 105^{\circ}, 120^{\circ}, 135^{\circ}, 150^{\circ}, 165^{\circ}$ e $180^{\circ}$, todas com as seguintes condições iniciais: semi-eixo maior: $1900000 \mathrm{~m}$, excentricidade: 0.001 , ascensão reta do nodo ascendente: $20^{\circ}$, argumento do periapside: $100^{\circ}$, anomalia média: $1^{\circ}$.

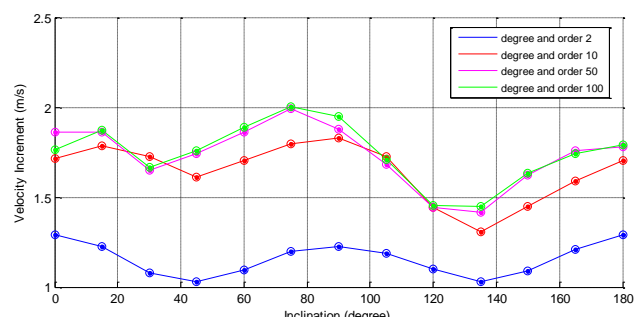

Figura 2: Variação de velocidade fornecida pela perturbação como função da inclinação da órbita do veículo

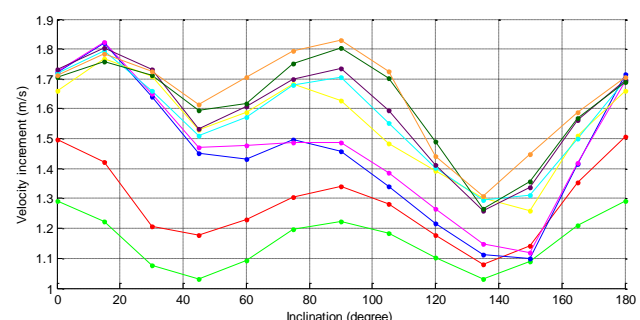

Figura 3: Variação de velocidade fornecida pela perturbação como função da inclinação da órbita do veículo espacial e do número de termos considerados para o potencial lunar para os 10 primeiros termos

Podemos notar uma diferença significativa quando se considera apenas grau e ordem 2 e quando os termos são considerados até grau e ordem 10, 50 e 100. Também é notado que existem valores de inclinação em que a perturbação gerada pelo potencial gravitacional lunar é maior. Analisando o caso com mais termos (grau e ordem 100), conclui-se que a inclinação de $75^{\circ}$ tem um valor mais alto para o incremento de velocidade e inclinação de $135^{\circ}$ tem o menor valor para o incremento da velocidade, com a diferença entre eles de aproximadamente 0,55 $\mathrm{m} / \mathrm{s}$, o que é muito significativo na escala de valores obtida, ou seja, cerca de $25 \%$. Isso significa que a inclinação desempenha um papel importante nas perturbações atuantes sobre pelo satélite.

Nota-se também que as simulações realizadas com 50 e 100 termos do potencial são semelhantes, enfatizando assim a pequena contribuição dos termos entre 50 e 100, como esperado. Mesmo considerando apenas os 10 primeiros termos, os resultados já são semelhantes aos resultados dos casos entre 50 e 100, o que demonstra o forte domínio dos 10 primeiros termos. Porém, há diferenças significativas ao se considerar apenas o primeiro termo e os outros 10 termos.

Assim, podemos ver na Figura 3 , o incremento de velocidade sobre satélite, devido ao potencial gravitacional para os 10 primeiros termos dos harmônicos esféricos, a fim de analisar a contribuição dos termos mais relevantes.

Na Figura 3, a linha verde representa o estudo realizado para grau e ordem 2, a linha vermelha para grau e ordem 3, a linha azul para grau e de ordem 4, a linha magenta para grau e ordem 5, a linha amarela para grau e ordem 6 , a linha azul clara para grau e ordem 7 , a linha roxa para grau e ordem 8 , a linha verde escura para grau e ordem 9 e linha laranja para grau e ordem 10.

\subsection{Manobras orbitais}

O estudo comparativo a seguir tem por objetivo analisar a influência do potencial gravitacional na órbita de um satélite artificial. Para esta tarefa, foram utilizadas como exemplo quatro simulações com a realização de manobras de correção e de transferência cujas condições 
iniciais são: semi-eixo maior: $2600000 \mathrm{~m}$ para as simulações 1 e 2 e $1900000 \mathrm{~m}$ para a simulações 3 e 4, e para as duas simulações excentricidade: 0.001 , inclinação: $45^{\circ}$, ascensão reta do nodo ascendente: $20^{\circ}$, argumento do periapse: $100^{\circ}$, anomalia média: $1^{\circ}$. Nas simulações $1 \mathrm{e}$ 3 são realizadas apenas manobras de transferência, e nas simulações 2 e 4 são realizadas manobras de transferência e correção.

As Figuras 4 e 5 mostram que o objetivo das simulações foi atingido com sucesso, uma vez que o semi-eixo maior diminuiu para simulações 1 e 2, e aumentou para simulações 3 e 4 . Em ambos os casos, o propulsor é ligado para realizar a órbita de transferência após o satélite completar uma órbita em torno da superfície da Lua, e é desligado quando o satélite atinge a altitude de $100 \mathrm{~km}$ para as simulações 1 e 2 e $815 \mathrm{~km}$ para a simulações 3 e 4 .

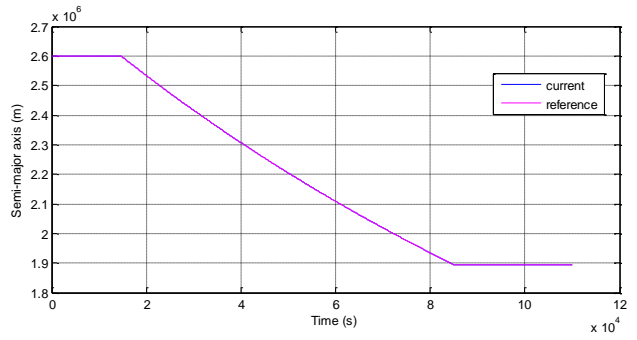

Figura 4: Semi-eixo maior durante as simulações 1 e 2

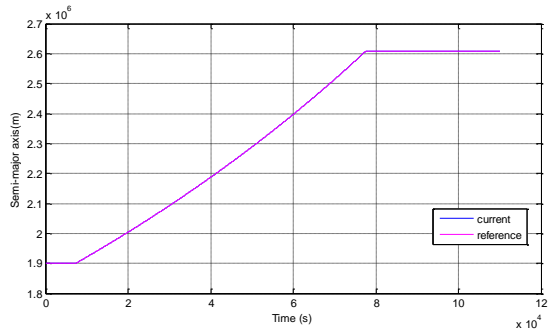

Figura 5: Semi-eixo maior durante as simulações 3 e 4

As Figuras 5 e 7 mostram a altitude do satélite e as Figuras 6 e 8 mostram a perturbação devido ao potencial gravitacional lunar.

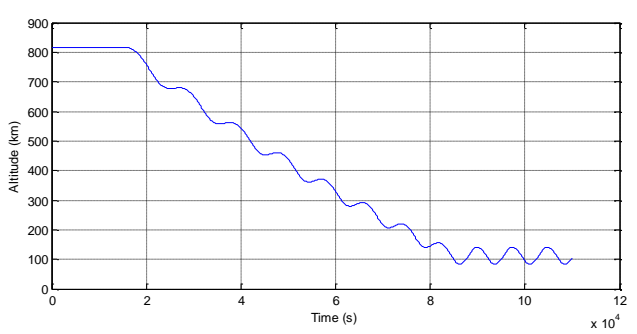

Figura 5: Altitude do satélite durante as simulações 1 e 2

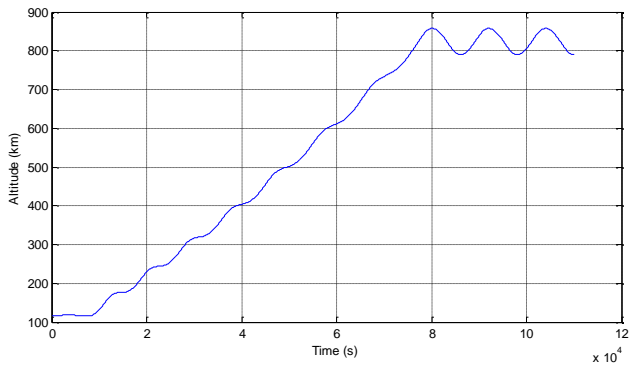

Figura 7: Altitude do satélite durante as simulações 3 e 4

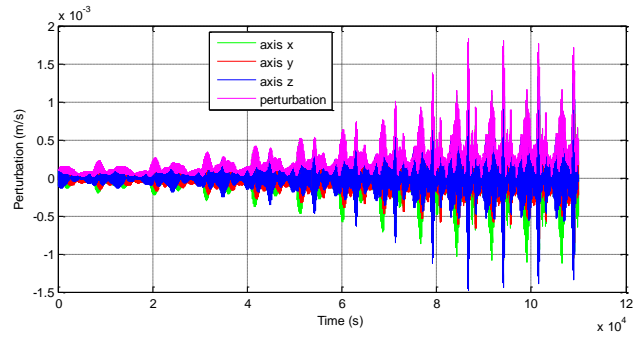

Figura 6: Perturbação sobre o satélite durante as simulações 1 e 2

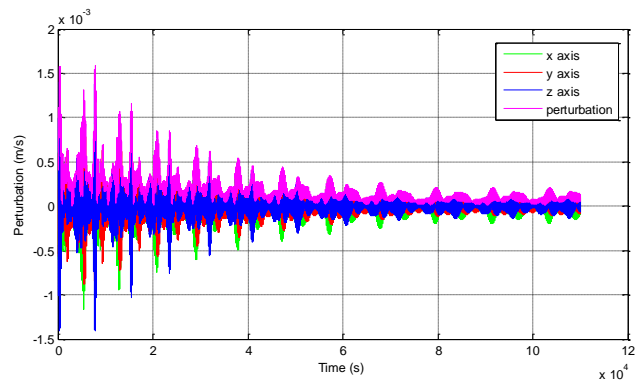

Figura 8: Perturbação sobre o satélite durante as simulações 3 e 4

De acordo com os objetivos das missões simuladas, podemos ver nas Figuras 5 e 7 as variações de altitude do satélite durante o tempo das simulações. O satélite teve uma variação de altitude de aproximadamente $715 \mathrm{~km}$, e oscilou em torno de $100 \mathrm{~km}$ de altitude para as simulações 1 e 2 e $830 \mathrm{~km}$ de altitude para simulações 3 e 4, uma vez que estes eram os objetivos da missão. Tais oscilações são decorrentes da excentricidade orbital. 
A partir das Figuras 6 e 8, vemos a força devido à perturbação do potencial gravitacional lunar aplicado sobre o satélite, isto é, a força de perturbação que tende a tirar o satélite da trajetória kepleriana. Podemos ver que o comportamento da perturbação não é constante, o que exige uma atuação intensa do sistema de controle.

Observa-se também que a intensidade de perturbação resultante sobre o satélite aumenta conforme o satélite se aproxima da superfície lunar, e diminui conforme o satélite se afasta da superfície lunar. É também visível que a perturbação estabiliza conforme a altitude também estabiliza.

Nas Figuras 8 e 9 podemos observar o empuxo aplicado ao satélite durante as simulações, com o objetivo de minimizar os efeitos causados pela perturbação, bem como realizar as manobras de transferência orbital. Vemos que o propulsor aplica força nos três eixos, ou seja, é possível que o sistema de controle atue em cada eixo separadamente.

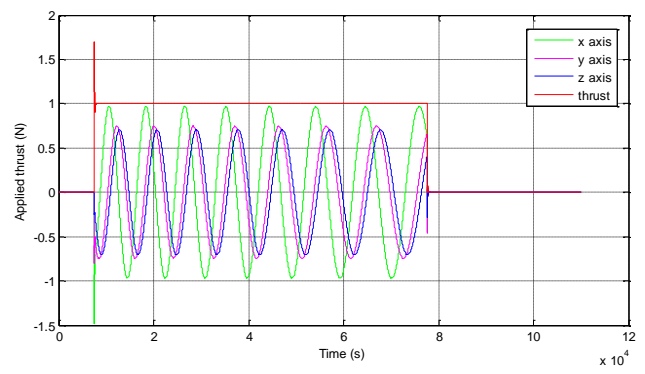

Figura 9: Empuxo aplicado ao satélite a cada instante de tempo durante as simulações 1 e 3

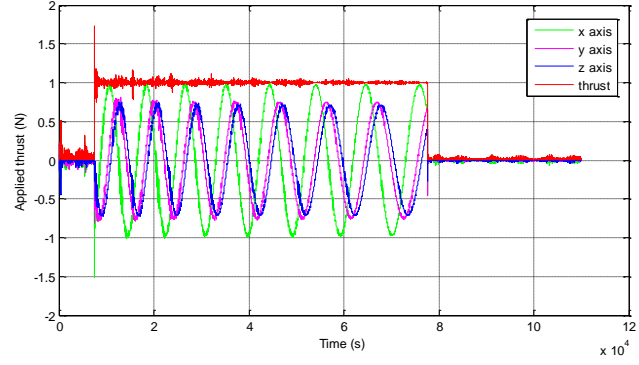

Figura 10: Empuxo aplicado ao satélite a cada instante de tempo durante as simulações 2 e 4

Nota-se nas Figuras 9 e 10 que, quando a manobra é finalizada, de acordo com as Figuras 5 e 7, a altitude atinge o valor especificado e tende a estabilizar. Neste momento, o propulsor é desligado. Também é possível observar que, devido a inconstância no comportamento do potencial gravitacional, como visto nas Figuras 6 e 8, é necessária uma atuação intensa do sistema de controle e consequentemente dos propulsores.

Sendo assim, o desempenho dos propulsores pode ser verificado para os dois casos em estudo: o caso em que manobras de correção e de transferência são realizadas simultaneamente e o caso em que apenas manobras de correção são realizadas para minimizar os efeitos perturbativos sobre o satélite. Para minimizar os efeitos do comportamento inconstante da perturbação, o sistema de controle atua sobre o satélite de forma intensa, como mostrado nas Figuras 6 e 8.

Os resultados das simulações mostram que existem diferenças entre a situação em que são realizadas apenas manobras de correção e a situação em que são realizadas manobras de correção e transferência. Para as simulações 1 e 2 , o caso em que as manobras de correção não são realizadas, o consumo de combustível é de cerca de $2,8685 \mathrm{~kg}$, e para o caso em que também são realizadas manobras de correção, o consumo é de $2,9273 \mathrm{~kg}$. Isso significa que uma diferença de $0,0588 \mathrm{~kg}$ de massa de propelente. Para as simulações 3 e 4 , o consumo para o primeiro caso é de $2,8641 \mathrm{~kg}$ e para o segundo caso é de 2,8953 , sendo a diferença massa de propelente consumida de $0,0312 \mathrm{~kg}$.

Esta pequena diferença ocorre porque, além de existir combustível consumido para a realização da manobra de transferência, ainda há um consumo de combustível utilizado para minimizar os efeitos da perturbação sobre a trajetória do satélite artificial.

\section{Conclusões}

Os resultados deste estudo são consistentes com o modelo apresentado por Konopliv (2001), que mostra a relação entre a aceleração gravitacional lunar e a topografia da Lua , mostrando diferença inconstante da aceleração da gravidade, devido à distribuição não uniforme da massa de a Lua, quando considerados diferentes valores para grau e ordem. 
Os estudos realizados e analisados com respeito aos efeitos de cada termo do potencial gravitacional na órbita de um satélite lunar artificial mostraram que o primeiro termo (grau e ordem 2) domina o movimento, mas as contribuições dos outros termos são relevantes, especialmente os primeiros 10 termos. Termos superiores a 10 são responsáveis por contribuições menores.

Os valores negativos aparecem neste estudo, demonstrando que alguns termos atuam na direção oposta à resultante das forças, ajudando a manter a órbita do satélite artificial perto de uma órbita kepleriana.

O estudo dos diferentes valores para a inclinação mostrou que a variação da inclinação pode alterar a perturbação sofrida pelo satélite artificial de forma significativa, mostrando que tais possibilidades devem ser levadas em consideração ao se planejar uma missão.

Estes estudos mostram que a perturbação em um satélite artificial devido à distribuição não uniforme da massa da Lua é significativamente inconstante, requerendo um intenso desempenho do sistema de controle para atenuar os desvios provocados na trajetória.

\section{Referências}

[1] Carvalho, J. P. S.; Moraes, R. V; Prado, A. F. B. A. Semi-analytic theory of a moon artificial satellite considering lunar oblateness and perturbations due to a third-body in elliptic orbit. In: Brazilian Conference on Dynamics Control, and Aplications, 7, 2008, Presidente Prudente. Proceedings...Presidente Prudente, 2008.

[2] Carvalho, J. P. S. Moraes, R. V; Prado, A. F. B. A. Non-sphericity of the moon and critical inclination. In: Congresso Nacional de Matemática Aplicada e Computacional, 32, 2009, Cuiabá, Brasil. Proceedings. Cuiabá, 2009(a).

[3] Carvalho, J. P. S. Moraes, R. V.; Prado, A. F. B. A. Non-sphericity of the Moon and near Sun-synchronous polar lunar orbits, Mathematical Problems in Engineering Article ID 740460, 24 pages. doi:10.1155/2009/740460, 2009(b).

[4] Carvalho, J. P. S. Moraes, R. V.; Prado, A. F. B. A. Some orbital characteristics of lunar artificial satellites, Celestial Mechanics \& Dynamical Astronomy, v. 108, n. 4, p. 371-388, DEC 2010.

[5] Gonçalves, L. D. Manobras Orbitais de Satélites Artificiais Lunares com Aplicação de Propulsão Contínua. 2013 - Instituto Nacional de Pesquisas Espaciais (INPE), São José dos Campos. 2013.

[6] Konopliv, A. S.; Asmar, S. W.; Carranza, E.; Sjogren, W. L.; Yuan, D. N. Recent gravity models as a result of the lunar prospector mission, Icarus, Vol. 150, pp. 1-18, Academic Press, 2001.

[7] Kuga, H.K.; Carrara, V.; Kondapalli R. R. Satélites Artificiais - Movimento Orbital. INPE - São José dos Campos, 2011. 111 p.

[8] Prado, A.F.B.A., Searching for Orbits with the Minimum Fuel Consumption for StationKeeping Maneuvers: Application to Luni-Solar Perturbations. Mathematical Problems in Engineering (Print), Volume 2013(2013), Article ID 415015, 11 pages.

[9] Ramanan, R. V.; Adimurthy, V. An analysis of near-circular lunar mapping orbits. J. Earth Syst. Sci. 114, No. 6, December 2005, pp. 619-626.

[10] Rocco, E. M. Perturbed orbital motion with a PID control system for thetrajectory. In: Colóquio Brasileiro de Dinâmica Orbital, 14, Águas de Lindóia,2008. Resumos...2008.

[11] Rocco, E. M. Controle de trajetória com propulsão contínua para missões do tipo dragfree. In: Congresso Nacional de Engenharia Mecânica, 7, 2012, São Luís, Brasil. Proceedings. São Luís, 2012. 